\title{
Development of Standard Solutions CAI System with UML and XML
}

\author{
Bojun Yang, Jianhui Zhang, Runhua Tan, Yumei Tian, Jianhong Ma \\ Hebei University of Technology, Institute of Design for Innovation, \\ Dingzigu, Hongqiao District, Tianjin, 300130, P.R.China \\ ybj@hebut.edu.cn \\ WWW home page: http://www.triz.com.cn
}

\begin{abstract}
CAI software products based on TRIZ were successfully applied in superior firms in the world. The Standard Solutions CAI system is one of branch of CAI software. Standard Solutions is important tool for product design. Standard Solutions for innovation firstly models a technical or process problem by Substance-Field (Su-F) Analysis, then synthesizes and converts the problem to acquire a solution. This paper researched on Standard Solutions arithmetic which can be applicable in CAI software product based on Standard Solutions, and established the flow of software. During the development of CAI software product, static models built based on UML were used to construct the configuration of system. For the components of system, dynamic models were used to describe the behaviors of system components. The Standard Solutions CAI software was coded with $\mathrm{VC}++$ language. The interfacial view of this software, which is friendly for users, was created by $\mathrm{VC}++$ combines with XML+XSL. Standard Solutions have great help to innovation design, farther more, its CAI software system help designer adequately apply the theory of Standard Solutions.
\end{abstract}

Key words: TRIZ, Standard Solutions, Computer-Aided Innovation, UML, XML

\section{Introduction}

Competition is the main impetus of technology progresses, also the resource of survival and development for enterprises. The contents of competition are change with varied time. Nowadays, most enterprises focus their attention on product design except for product qualities, cost, and production process and so on. Various methodologies and methods of product design progress quiet great recently years, like TRIZ, AD, QFD and TOC. At the same time, information technology applied

Please use the following format when citing this chapter:

Yang, B., Zhang, J., Runhua, T., Tian, Y., Ma, J., 2007, in IFIP International Federation for Information Processing, Volume 250, Trends in Computer Aided Innovation, ed. León-Rovira, N., (Boston: Springer), pp. 157-165. 
widely to product design stage, from CAD and FEA, to CAI. Information technology is fusing in design methods more and more.

In the former Soviet Union, TRIZ [1], the theory of Inventive Problem Solving, was developed by Genrikh Altshuler and his school beginning in 1946. After more than 60 years development and application, TRIZ was proved powerful for concept generation and innovation design. Standard Solutions is one TRIZ knowledge of many parts and kinds of TRIZ. Standard Solutions include 76 items, can be used to solve relatively common optimization problems, and more particular to solve level three inventive problems [2].

The aim of Computer Aided Innovation (CAI) is help product designer can use innovative theory more effective and get more doable product design scheme. CAI software have many kinds, but TRIZ-based software is mainstream and extensive. From 1991, a TRIZ-based software package which developed by the Invention Machine Corporation was commercially launched [3], many CAI software based TRIZ have been developed now, such as TRISolver, IWB, Goldfire and so on.

To using Standard Solutions, this paper research on develop a CAI software system based on Standard Solutions. The system is a module of InventionTool3.0.

\section{Standard Solutions and Theirs Usage}

\subsection{Substance-Field}

According to the process of inventive problem solving, TRIZ system constructed from the theoretical foundation, laws of evolution, the analytical tools, such as contradiction analysis and required function analysis, and knowledge base tools, such as 40 principles and effects database. Substance-Field analysis, simply, Su-F analysis, is one of analytical tools or Search engines, and Standard Solutions is knowledge base tool (Table 1).

Table 1. The knowledge to support inventive problem solving

\begin{tabular}{lll}
\hline Knowledge & Search engine & Levels of solutions \\
\hline 40 principles & 39 problem parameters and Matrix & 2 \\
76 Standard soluivis & Su-ficld Nodel & $2,3,4$ \\
Effects & 30 TRIZ standard functions & $2,3,4$ \\
TE patterns and paths & TE patterns & $2,3,4$ \\
\hline
\end{tabular}

Su-F is a graphical model of a minimal working technique in TRIZ [4]. Figure 1 is a simple description of Su-F Model. Su-F Model provide a fast, simple description of subsystems and their interaction in a technical system via a well-formulated model of the technique in which all subsystems, inputs, and outputs are known or can be quite easily determined. Su-F Model is one of the most important analysis tools in TRIZ, usually used in ARIZ with Standard Solutions.

The use of the Standard Solutions begins with abstracting an initial Su-F model (ISM) from a freely worded description of a problem or situation. Generally, Su-F analysis is adapts to any situation, and figure 2 shows the analysis process [3]. 
According above, the CAI system based on Standard Solutions should include the Substance-Field Model.

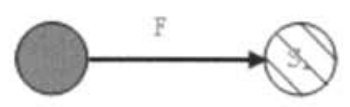

Fig. 1. A simple Su-F Model

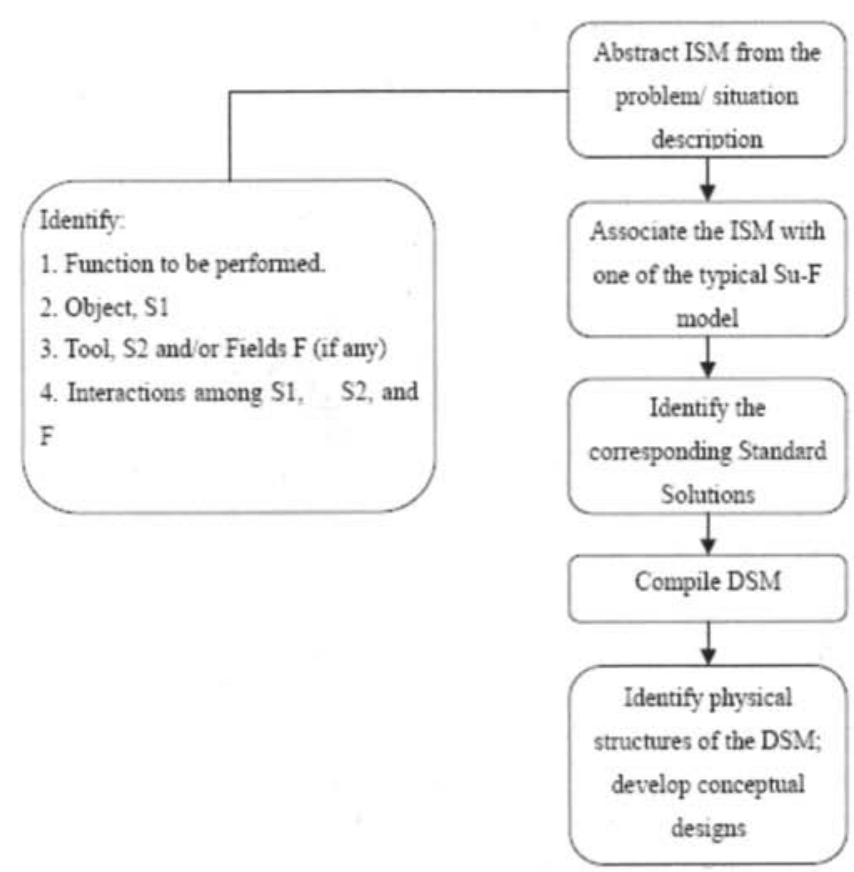

Fig. 2. Building Su-F Model using the Standard Solutions [3]

If a technology system, which needs a certain improvement, is modeled as a typical Su-F model, then a corresponding typical transformation can be used to improve the system. Typical Su-F model transformations are called Standard Solutions to solving Problems or, simply, the Standards [3]. Altshuller discovered various engineering problem from different domains can be solved by generic conceptual approaches, which is Standards. They are grouped into 5 or 3 large classes as Table 2.

The 76 Standard Solutions are used to solve relatively common optimization problems. Further more, they are useful for level three inventive problems. Typically, the Standard Solutions are used as a step in ARIZ, after the Su-F Model has been developed and any constraints on the solution have been identified. 
Standards and Su-F are linking tightly each other. Before use Standards, a Su-F Model of technical problem must be constructed mostly.

Table 2. The Standard Solutions categorization

\begin{tabular}{|c|c|c|}
\hline 1. Improving the system with no or little change & & 13 \\
\hline 2. Improving the system by changing the system & System modification & 23 \\
\hline 3. System transitions & & 6 \\
\hline 4. Detection and measurement & System detection and measuring & 17 \\
\hline 5. Strategies for simplification and improvement & Application of Standards & 17 \\
\hline & Total: & 76 \\
\hline
\end{tabular}

\subsection{Algorithms for using the Standard Solutions}

Standards are the precepts of synthesis and transformation with the aim to overcome or circumvent technical and physical contradictions [4]. According to figure 2, the Standard Solutions are typical Su-F model transformations. When the ISM's have been built, they must be associated with the available typical Su-F models. Then these Su-F models must be related to one or more of Standard Solutions that "prescribe" transition to more advanced, desirable Su-F models (DSMs). During this process, the appropriate Standard Solutions should be choosing. Some algorithms for choosing the right Standards had been designed.

Joe Miller and Ellen Domb have developed a flowchart for Using the 76 Standard Solutions in the Proceedings of TRIZCON2001. The flowchart organizes use of the 76 Standard solutions into three main pathways: system improvement, solutions for Measurement and Detection, and use of the Standard Solutions for Forecasting opportunities for change [5]. This flowchart is helpful for designer to comprehend the structure of Standard Solutions System. Designer can get a suggestion of class or group level using this flowchart.

Reference 3 thought the Standards are organized in a set built according to the logic of evolution of Su-F models: simple Su-F models $\rightarrow$ complex Su-F models $\rightarrow$ intensified Su-F models [3]. So, it provided an algorithm flowchart organized with the logic. This algorithm deal with initial Su-F models in distinguishing detection/measuring problem and non-detection/measuring problem. Based on the transform of Su-F model, user can obtains a right Standard Solution.

Another detailed algorithm was mentioned in reference 4. That algorithm is more detailed and effective than above for choose each item of Standard Solutions. It begin with construct a model of the problem, through a series of opinion according constraints or restrictions, then confirm one or more Standard Solutions for solving problem.

\subsection{An applicable algorithm for CAI software}

All above of the algorithms or methods is useful for designer when they use Standard Solutions to solve innovative problem. When a CAI system based on Standard Solutions was developed, those algorithms must be adjusted to adapt to 
computer environment. We designed a new algorithm that integrated the advantages of previous and was suitable for computer procedure. Its simplified flowchart shows in figure 3.

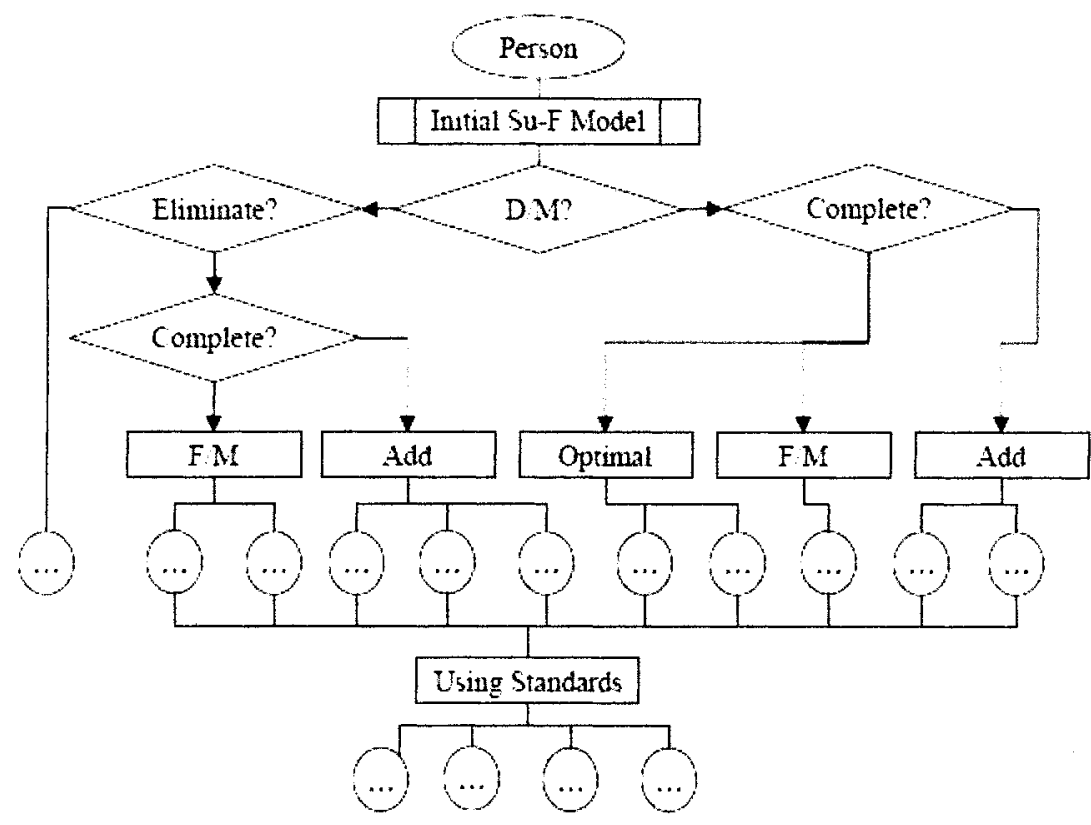

Fig. 3. The simplified flowchart of algorithm using in CAI system

This algorithm has some features:

a) Description of constraints is exact;

b) Cover with all the 76 Standard Solutions;

c) Arrangement of group or class or item is clear;

\section{The CAI System Based on Standard Solutions}

\subsection{Situation of CAIs Based on TRIZ}

Computer Aided Innovation software based on innovational theory is a kind of CAD that is suitable for conceptual design stage [6]. For TRIZ has systematized configuration, multi tools to analyze and model problem, mighty knowledge database to provide example or analogy cases, TRIZ-based CAI software have been developed rapidly and widely than other innovational theory.

In 1991, a TRIZ-based software package had been developed by the Invention Machine Corporation. TRISolver $2.1^{\mathrm{TM}}$ is the early English-language version of the 
CAI software from TRISolver Consulting Group in Germany. The Invention Machine in America had issued TechOptimizer, and than upgrade to Goldfire Innovator. Another America firm Ideation International developed the Innovation WorkBench (IWB) and so on. Those English-language software of CAI integrate the concepts, principles and tools of TRIZ. Those CAI software were applied in practice and made great success ${ }^{7}$. In China, InventionTool had been commercially launched by the $3^{\text {rd }}$ version.

Some specialized functional CAI system were developed depend upon TRIZ tools, such as inventive principles, technology evolution, effect, and technology maturation forecast and so on. Usage of them can make designer agile, convenient and quick solve some little problem. Those systems have growing potentiality.

\subsection{The Structure of Standard Solutions CAI System}

Basing on the above algorithm for using Standard Solutions, a Standard Solutions CAI system is developed. In the CAI system it is realized not only to support technical knowledge but to solve problem using in Standard Solutions systemically.

Standard Solutions CAI system is a module of InventionTool CAI software, also can be used individual. TRIZ is innovational theory based on knowledge, and same to the Standard Solutions as a part of TRIZ. The whole system should include four components: Substance-Field, Standard Solutions, Algorithm and database, as shown in figure 4.

Before using the Standard Solutions to solve problem, designers should be expert in modeling by Su-F and in all the Standards. In the system, Substance-Field component show the definition and graphic representation for designer. Standard Solutions component enumerate items one by one with examples. Algorithm provides the process solving problem using Standards. Database stores the examples which can be analogized, and it can be add new examples.

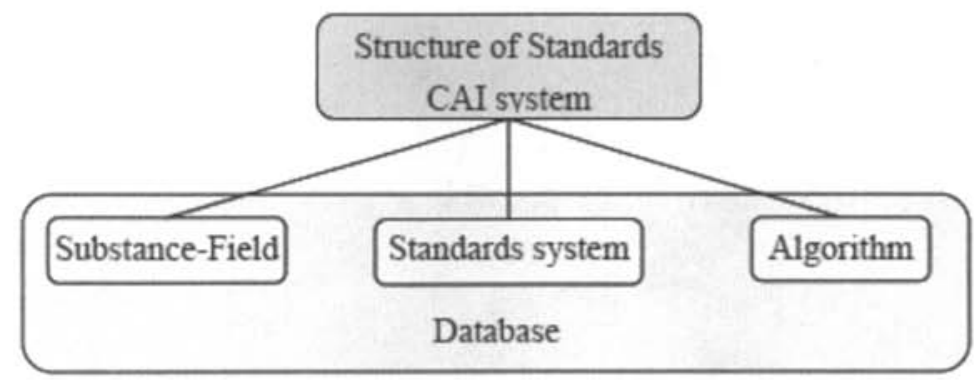

Fig. 4. Structure of Standard Solutions CAI System 


\subsection{Method and Tools Used in Software Development}

In term of the lifecycle of software, a software development process is fallen into 6 steps: analysis, design, implementation, module test, system test and executing. The Standard Solution system follows the same process. Object-Oriented method [8] is the mainstream method to develop software. UML method integrated and expended the advanced characteristics of some other Object-Oriented methods. The system analysis obeys UML, and implement with VC++ language and XML.

The Unified Modeling Language (UML) is a modeling language for specifying, visualizing, constructing, and documenting the artifacts of a system-intensive process. [9] Before to program codes, the models of software system can help software designer study the various constructions and schemes conveniently. Through construct a series of models or diagrams, "what" is required of a system, and "how" a system may be realized was shows before software designers. .UML is applicable in different stages of system development, from description of required specification to system test and maintenance.

After analyzing the Standard Solutions CAI system, software was coded with $\mathrm{VC}++$ language. The interfacial view of this software, which is friendly for users, was created by $\mathrm{VC}++$ combines with $\mathrm{XML}+\mathrm{XSL}$. For the CAI system has a large database, XSL is a suitable tool to deal with data transfer and data store. XML (eXtensible Markup Language) is used to develop the interface of the software.

\section{UML Models in the Standards CAI System}

During the development of CAI software product, static models such as Use Case Diagram, Class Diagram, Object Diagram, Package, Component Diagram and Deployment Diagram built based on UML were used to construct the configuration of system. For the components of system, dynamic models such as State Diagram, Sequence Diagram, Collaboration Diagram and Activity Diagram were used to describe the behaviors of system components.

Before develop the Standard Solutions CAI system, Use Case model should be construct, like shows in figure 5. It shows what is actor or user recognized of system and how to operate it. 


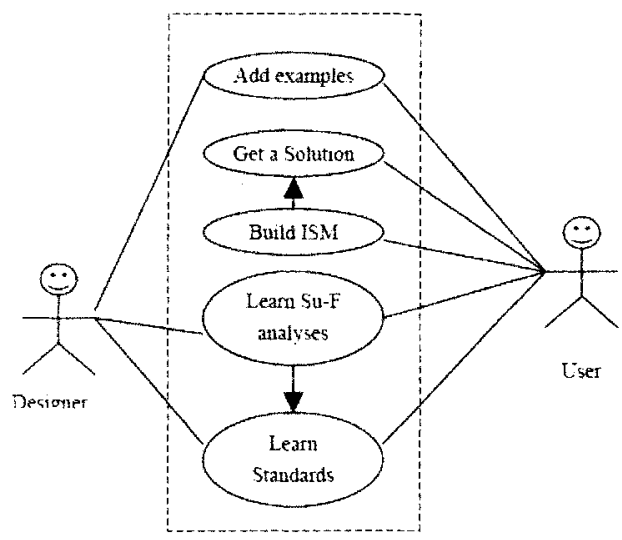

Fig. 5. The Use Case Diagram of Standards CAI system

During the software was programmed, many kinds of UML model had been built. They show different relationships and operation. Figure 6 is a Activity model which shows add new examples to system. The activity of add was shown clearly.

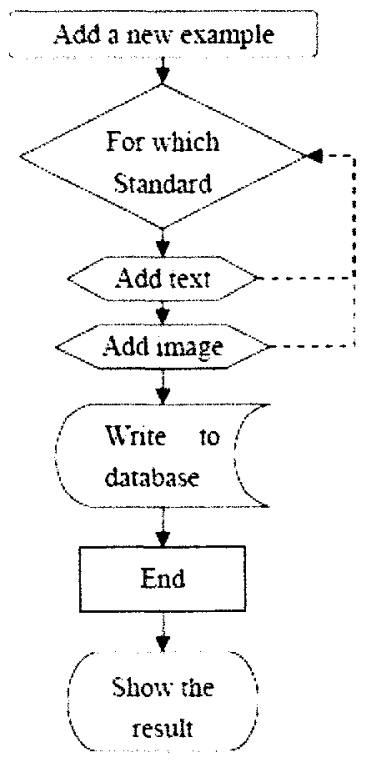

Fig. 6. The Activity Diagram of add new example to system

All above of the UML model is helpful to develop software, improve the efficiency of programming. 


\section{Conclusions}

Standard Solutions for innovation firstly models a technical or process problem by Substance-Field (Su-F) Analysis, then synthesizes and converts the problem to acquire a solution. A CAI system based on Standard Solutions was developed that include Su-F model, Standards and theirs usage. The algorithm for using Standard Solutions was improved. The development supported by UML method, $\mathrm{VC}++$ language and XML. Convenient usage and friendly interface have great help to innovation design. The method for development of CAI product is instructive exploration which had been proved to be efficient and feasible during the process of practice.

\section{Acknowledgement}

The research is supported in part by the Chinese Natural Science Foundation under Grant Numbers 50675059 and Chinese national 863 planning project under Grant Number 2006AA04Z109.

\section{References}

1. Altshuller G., The Innovation Algorithm, TRIZ, systematic innovation and technical vreativity, Technical Innovation Center, INC, Worcester, 1999

2. John Terninko, Ellen Domb, Joe Miller, The Seventy-six Standard Solutions with Examples, The TRIZ journal, February, 2000, http://www.triz-journal.com.

3. Victor Fey and Eugene Rivin, Innovation on Demand, New Product Development Using TRIZ, Cambridge University Press, 2005

4. Savransky S D, Engineering of Creativity, New York: CRC Press, 2000

5. Joe Miller, Ellen Domb, Ellen MacGran, John Terninko, Using the 76 Standard Solutions: A case study for improving the world food supply, The TRIZ journal, April, 2001, http://www.triz-journal.com.

6. Tan Runhua, Progress of some problems in product design for innovation, Chinese Journal of Mechanical Engineering, Sep., 2003

7. Yang Bojun, Tan Runhua, Tian Yumei, Development of a CAI system of Standard solutions based on TRIZ, Proceedings of PROLAMAT2006, IFIP TC5 International Conference, Shanghai, 2006

8. Hassan Gomaa, An Object-Oriented domain ananlysis and modeling method for software reuse, 0073-1129-1/92, 1992 IEEE

9. Grady Booch, James Rumbaugh, Ivar Jacobson. The Unified Modeling Language User Guide. Addison- Wesley, Reading, Mass, 1999 